\title{
Universal Algorithm for Optimal Estimation of Quantum States from Finite Ensembles
}

\author{
R. Derka ${ }^{1,2}$, V. Bužek ${ }^{2,3}$, and A.K. Ekert ${ }^{1}$ \\ 1 Department of Physics, Oxford University, Parks Road, OX1 3PU Oxford, U.K. \\ 2 Institute of Physics, Slovak Academy of Sciences, Dúbravská cesta 9, 84228 Bratislava, Slovakia. \\ ${ }^{3}$ Optics Section, The Blackett Laboratory, Imperial College, London SW' 2BZ, U.K.
}

(13 July 1997)

We present a universal algorithm for the optimal quantum state estimation of an arbitrary finite dimensional system. The algorithm specifies a physically realizable positive operator valued measurement (POVM) on a finite number of identically prepared systems. We illustrate the general formalism by applying it to different scenarios of the state estimation of $N$ independent and identically prepared two-level systems (qubits).

Suppose we have $N$ quantum objects, each prepared in an unknown pure quantum state described by a density operator $\hat{\rho}=|\psi\rangle\langle\psi|$. What kind of measurement provides us with the best possible estimation of $\hat{\rho}$ ?

Clearly, if we have an unlimited supply of particles in state $\hat{\rho}$, i.e. when $N$ approaches infinity, we can estimate $\hat{\rho}$ with an arbitrary precision. In practice, however, only finite and usually small ensembles of identically prepared quantum systems are available. This leads to an important problem of the optimal state estimation with fixed physical resources.

A particular version of this problem has recently been addressed by Massar and Popescu [1] who have analyzed extraction of information from finite ensembles of spin$1 / 2$ particles. They have shown that there exists a fundamental limit on the fidelity of estimation and they have constructed a POVM consisting of an infinite continuous set of operators which attains this limit. It has been suggested that the optimal finite POVM may exist, however, so far no explicit construction of such finite POVM has been provided. We solve this problem by describing an algorithm which gives the optimal and finite POVM. We also provide a concise derivation of a general bound on the fidelity of a state estimation of arbitrary finitedimensional systems (the Massar-Popescu bound follows as a particular case). Finite POVMs can be, at least in principle, constructed and the optimal state estimation procedures may be applied in many areas of physics ranging from the optimal phase estimation to quantum computation.

In fact, in the following we are solving a more general problem of optimal estimation of unitary operations performed on quantum objects (the state estimation follows as a special case). Let us assume that state $\hat{\rho}$ is generated from a reference state $\hat{\rho}_{0}=\left|\psi_{0}\right\rangle\left\langle\psi_{0}\right|$ by a unitary operation $U(\mathbf{x})$ which is an element of a particular unitary representation of some group G. Different $\mathbf{x}$ denote different points of the group (e.g., different angles of rotation in the case of the $S U(2))$ and we assume that all values of $\mathbf{x}$ are equally probable.

Our task is to design the most general POVM, mathematically described as a set $\left\{\hat{O}_{r}\right\}_{r=1}^{R}$ of positive Hermitian operators such that $\sum_{r} \hat{O}_{r}=\hat{1}$ [2, 3], which when applied to the combined system of all $N$ copies provides us with the best possible estimation of $\hat{\rho}$ (and therefore also of $U(\mathbf{x}))$. We quantify the quality of the state estimation in terms of the mean fidelity

$$
\begin{aligned}
\bar{f} & =\sum_{r} \int_{G} d \mathbf{x} \operatorname{Tr}[\hat{O}_{r} \overbrace{U(\mathbf{x}) \hat{\rho}_{0} U^{\dagger}(\mathbf{x}) \otimes \ldots \otimes U(\mathbf{x}) \hat{\rho}_{0} U^{\dagger}(\mathbf{x})}^{\mathbf{N} \text { times }}] \\
& \times \operatorname{Tr}\left[U(\mathbf{x}) \hat{\rho}_{0} U^{\dagger}(\mathbf{x}) U_{r} \hat{\rho}_{0} U_{r}^{\dagger}\right]
\end{aligned}
$$

which corresponds to a particular choice of a cost function [3] used in a context of detection and estimation theory. The mean fidelity (11) can be understood as follows: In order to assess how good a chosen measurement is we apply it many times simultaneously on all $N$ particles each in state $U(\mathbf{x}) \hat{\rho}_{0} U^{\dagger}(\mathbf{x})$. The parameter $\mathbf{x}$ varies randomly and isotropically [1] over all points of the group $G$ during many runs of the measurement.

For each result $r$ of the measurement, i.e., for each operator $\hat{O}_{r}$, we prescribe the state $\left|\psi_{r}\right\rangle=U_{r}\left|\psi_{0}\right\rangle$ which represents our guess (i.e., estimation) of the original state. The probability of the outcome $r$ is equal to $\operatorname{Tr}\left[\hat{O}_{r} U(\mathbf{x}) \hat{\rho}_{0} U^{\dagger}(\mathbf{x}) \otimes \ldots \otimes U(\mathbf{x}) \hat{\rho}_{0} U^{\dagger}(\mathbf{x})\right]$ while the corresponding fidelity of the state estimation is $\left|\left\langle\psi_{r} \mid \psi\right\rangle\right|^{2}=$ $\operatorname{Tr}\left[U(\mathbf{x}) \hat{\rho}_{0} U^{\dagger}(\mathbf{x}) U_{r} \hat{\rho}_{0} U_{r}^{\dagger}\right]$. This fidelity is then averaged over all possible outcomes and over many independent runs of the measurement with randomly and isotropically distributed parameters $\mathbf{x}$. We want to find the generalized measurement which maximizes the mean fidelity $\bar{f}$ given by Eq.(11).

The combined system of $N$ identically prepared reference states always remains within the totally symmetric subspace of $H^{k} \otimes H^{k} \otimes \ldots H^{k}$, where $H^{k}$ is $k$-dimensional Hilbert space of the reference state in which the corresponding unitary representation $U(\mathbf{x})$ acts. Thus the dimensionality $d$ of the space in which we construct the POVM $\left\{\hat{O}_{r}\right\}$ is $d=\left(\begin{array}{l}N+k-1 \\ k-1\end{array}\right)$. In this case the first trace in Eq.(1) can be rewritten as 


$$
\begin{aligned}
\bar{f} & =\sum_{r} \int_{G} \operatorname{Tr}\left[\hat{O}_{r} U^{N}(\mathbf{x}) \hat{\Omega}_{0} U^{N \dagger}(\mathbf{x})\right] \\
& \times \operatorname{Tr}\left[U(\mathbf{x}) \hat{\rho}_{0} U^{\dagger}(\mathbf{x}) U_{r} \hat{\rho}_{0} U_{r}^{\dagger}\right] d \mathbf{x},
\end{aligned}
$$

where $U^{N}(\mathbf{x})$ is a new representation of the same group $G$; it is equivalent to the $N$-fold symmetrized direct product [5] of the original representation $U(\mathbf{x})$. Here $U^{N}(\mathbf{x})$ transforms the $\left(\begin{array}{l}N+k-1 \\ k-1\end{array}\right)$-dimensional reference state denoted as $\hat{\Omega}_{0}$.

We can insert the identity operator $U_{r}^{N} U_{r}^{N \dagger}$ into the first trace in Eq. (2) and, taking into account that in Eq.(22) we integrate over whole the group $G$ parameterized by $\mathbf{x}$, we can substitute $U^{N}(\mathbf{x}) U_{r}^{N \dagger} \rightarrow U^{N}(\mathbf{x})$ and $U(\mathbf{x}) U_{r}^{\dagger} \rightarrow U(\mathbf{x})$. Now, using the linearity of the trace operation as well as the linearity of the representation of the group $G\left(U \hat{\rho} U^{\dagger}\right.$ is a linear adjoint representation) we rewrite Eq.(2) as

$$
\bar{f}=\sum_{r} \operatorname{Tr}\left[\hat{O}_{r} U_{r}^{N} \hat{F} U_{r}^{N \dagger}\right],
$$

where

$$
\hat{F}=\int_{G} U^{N}(\mathbf{x}) \hat{\Omega}_{0} U^{N \dagger}(\mathbf{x}) \operatorname{Tr}\left[U(\mathbf{x}) \hat{\rho}_{0} U^{\dagger}(\mathbf{x}) \hat{\rho}_{0}\right] d \mathbf{x},
$$

is a positive Hermitian operator.

Let us now derive an upper bound on the mean fidelity. Taking into account positivity of the operator $\hat{F}$ (i.e., $\left.\hat{F}=\sum_{i} \lambda_{i}\left|\phi_{i}\right\rangle\left\langle\phi_{i}\right| ; \quad \lambda_{i} \geq 0\right)$ and the completeness condition for POVM (i.e., $\sum_{r} \hat{O}_{r}=\hat{1}$ ) we obtain

$$
\begin{aligned}
\bar{f} & =\sum_{r} \operatorname{Tr}\left[\hat{O}_{r} U_{r}^{N} \hat{F} U_{r}^{N \dagger}\right]=\sum_{i r} \lambda_{i} \operatorname{Tr}\left[\hat{O}_{r} U_{r}^{N}\left|\phi_{i}\right\rangle\left\langle\phi_{i}\right| U_{r}^{N \dagger}\right] \\
& \leq \lambda_{\max } \sum_{i r} \operatorname{Tr}\left[\hat{O}_{r} U_{r}^{N}\left|\phi_{i}\right\rangle\left\langle\phi_{i}\right| U_{r}^{N \dagger}\right] \\
& =\lambda_{\max } \sum_{r} \operatorname{Tr}\left[\hat{O}_{r} U_{r}^{N} \hat{1} U_{r}^{N \dagger}\right]=\lambda_{\max } \operatorname{Tr}[\hat{1}]=\lambda_{\max } d .
\end{aligned}
$$

From Eq.(5) it clearly follows that the upper bound can be achieved if and only if all operators $\hat{O}_{r}$ forming the POVM satisfy the following conditions:

i) Each $\hat{O}_{r}$ is proportional to a suitably rotated (by some $U_{r}^{N}$ ) projector on the eigenvector of $\hat{F}$ with the highest eigenvalue, i.e. for all $\hat{O}_{r}$ there exists $U_{r}^{N}$, such that $\hat{O}_{r}=c_{r}^{2} U_{r}^{N}\left|\phi_{\max }\right\rangle\left\langle\phi_{\max }\right| U_{r}^{N \dagger}$. This $U_{r}^{N}$, or more precisely $U_{r}\left|\psi_{0}\right\rangle$, is our guess associated with the result " $r$ ". ii) All $c_{r}^{2}$ are real and positive, to assure that all $\hat{O}_{r}$ are positive operators.

iii) Finally, the operators $\hat{O}_{r}$ have to satisfy the completeness criterion $\sum_{r} c_{r}^{2} U_{r}^{N}\left|\phi_{\max }\right\rangle\left\langle\phi_{\max }\right| U_{r}^{N \dagger}=\hat{1}$.

However, these three conditions are not necessarily compatible (see Example B below). Therefore, in general, the solution cannot be found via this type of considerations and we have to proceed in a different way.
We start with the following observation. Let us assume that we have some POVM $\left\{\hat{O}_{r}\right\}_{r=1}^{R}$ and the corresponding guesses $U_{r}^{N}$ which maximize the mean fidelity $\bar{f}$. We can always construct another POVM with more elements which is also optimal. For example, let us consider a one-parametric subgroup $U(\phi)=\exp (i \hat{X} \phi)$ of our original group $G$ and choose a basis $\{|m\rangle\}_{m=1}^{d}$ in which the action of this subgroup is equivalent to multiplication by a factor $\mathbf{e}^{i \omega_{m} \phi}$ (i.e., the operator $U(\phi)$ is diagonal in this basis and $\omega_{m}$ are eigenvalues of the generator $\hat{X})$. Then we take $d$ points $\phi_{s}(s=1, \ldots d)$ and generate from each original operator $\hat{O}_{r}$ a set of $d$ operators $\hat{O}_{r s}=\frac{1}{d} U^{N}\left(\phi_{s}\right) \hat{O}_{r} U^{N \dagger}\left(\phi_{s}\right)$. In this way we obtain a new set of $(d \cdot R)$ operators such that the mean fidelity for this new set of operators, $\bar{f}=\sum_{r, s} \operatorname{Tr}\left[\hat{O}_{r, s} U_{r, s}^{N} \hat{F} U_{r, s}^{N \dagger}\right]$, is equal to the mean fidelity of the original POVM $\left\{\hat{O}_{r}\right\}$ because we ascribe to each eventual result $[r, s]$ a new guess $U_{r, s}=U\left(\phi_{s}\right) U_{r}$. However, in order to guarantee that the new set of operators $\hat{O}_{r s}$ is indeed a POVM we have to satisfy the completeness condition

$$
\begin{aligned}
\hat{1} & =\sum_{s} \sum_{r} \hat{O}_{r s}^{{ }^{\prime}}=\sum_{s} \sum_{r} \frac{1}{d} U^{N}\left(\phi_{s}\right) \hat{O}_{r} U^{N \dagger}\left(\phi_{s}\right) \\
& =\sum_{s} \sum_{m, n} \frac{\mathbf{e}^{i \phi_{s}\left(\omega_{m}-\omega_{n}\right)}}{d} \sum_{r}\left(\hat{O}_{r}\right)_{m n}|m\rangle\langle n| .
\end{aligned}
$$

Let us notice that, by the appropriate choice of $\phi_{s}$, the sum $\sum_{s} \frac{\mathbf{e}^{i \phi_{s}\left(\omega_{m}-\omega_{n}\right)}}{d}$ can always be made equal to $\delta_{m, n}$ providing all eigenvalues are non-degenerate [6] (this is basically a discrete Fourier transform and we illustrate this point in detail in Example A). In this case, the conditions (6) for the off-diagonal terms in the basis $|m\rangle$ are trivially satisfied whereas the diagonal terms are equal to unity because the original POVM $\left\{\hat{O}_{r}\right\}$ guarantees that $\sum_{r}\left(\hat{O}_{r}\right)_{m m}=1$. Moreover, even if the original set of operators $\left\{\hat{O}_{r}\right\}$ does not satisfy the full completeness condition and the conditions for the off-diagonal terms are not satisfied (i.e., these operators do not constitute a POVM) we can, using our extension ansatz, always construct a proper POVM $\left\{\hat{O}_{r, s}\right\}$. This proves that when we maximize the mean fidelity (3) it is enough to assume $d$ diagonal conditions rather than the original complete set of $d^{2}$ constraints for diagonal and off-diagonal elements.

Now we turn back to our original problem of how to construct the POVM which maximizes the mean fidelity. To do so we first express the operators $\hat{O}_{r}$ in the form $\hat{O}_{r}=c_{r}^{2} U_{r}^{N}\left|\Psi_{r}\right\rangle\left\langle\Psi_{r}\right| U_{r}^{N \dagger}$, where $\left|\Psi_{r}\right\rangle$ are general normalized states in the $d$-dimensional space in which the operators $\hat{O}_{r}$ act, and $c_{r}^{2}$ are positive constants. This substitution is done without any loss of generality [7] and it permits us to rewrite Eq.(3) so that the mean fidelity $\bar{f}$ does not explicitly depend on $U_{r}^{N}$, i.e.

$$
\bar{f}=\sum_{r} c_{r}^{2} \operatorname{Tr}\left[\left|\Psi_{r}\right\rangle\left\langle\Psi_{r}\right| \hat{F}\right] .
$$


Obviously, the completeness condition $\sum_{r} \hat{O}_{r}=\hat{1}$ is now modified and it reads

$$
\sum_{r} c_{r}^{2} U_{r}^{N}\left|\Psi_{r}\right\rangle\left\langle\Psi_{r}\right| U_{r}^{N \dagger}=\hat{1} .
$$

From our discussion above it follows that when maximizing the mean fidelity ( 7 it is enough to apply only $d$ constraints $\sum_{r} c_{r}^{2}\left|\left\langle m\left|U_{N_{,}}\right| \Psi_{r}\right\rangle\right|^{2}=1$ (here $m=1, \ldots, d$ ) out of the $d^{2}$ constraints (8). Therefore to accomplish our task we solve a set Langrange equations with $d$ Lagrange multipliers $L_{m}$. If we express $L_{m}$ as eigenvalues of the operator $\hat{L}=\sum_{m} L_{m}|m\rangle\langle m|$ then we obtain the final very compact set of equations determining the optimal POVM

$$
\left[\hat{F}-U_{r}^{N \dagger} \hat{L} U_{r}^{N}\right]\left|\Psi_{r}\right\rangle=0, \quad \sum_{r} c_{r}^{2}\left|\left\langle m\left|U_{r}^{N}\right| \Psi_{r}\right\rangle\right|^{2}=1 .
$$

From here it follows that $\left|\Psi_{r}\right\rangle$ are determined as zeroeigenvalue eigenstates. More specifically, they are functions of $d$ Lagrange multipliers $\left\{L_{m}\right\}_{m=1}^{d}$ and $R$ vectors $\left\{\mathbf{x}_{r}\right\}_{r=1}^{R}$ [where $\mathbf{x}_{r}$ determine $U_{r}$ as $U_{r}=U\left(\mathbf{x}_{r}\right)$ ]. These free parameters are in turn related via $R$ conditions $\operatorname{Det}\left[\left(\hat{F}-U_{r}^{N \dagger} \hat{L} U_{r}^{N}\right)\right]=0$. The mean fidelity now is equal to $\operatorname{Tr} \hat{L}$. At this stage we solve a system of $d$ linear equations [see the second formula in Eq.(9)] for $R$ unknown parameters $c_{r}^{2}$. All solutions for $c_{r}^{2}$ parametrically depend on $L_{m}$ and $\mathbf{x}_{r}$ which are specified above. We note that the number of free parameters in our problem depends on $R$ which has not been specified yet. We choose $R$ such that there are enough free parameters so that the mean fidelity is maximized and simultaneously all $c_{r}^{2}$ are positive. This freedom in the choice of the value of $R$ also reflects the fact that there is an infinite number of equivalent (i.e., with the same value of the mean fidelity) optimal POVMs. The whole algorithm is completed by finding $\phi_{s}$ from Eq.(6) which explicitly determine the finite optimal POVM $\left\{\hat{O}_{r s}\right\}$. This is the main result of our Letter.

In the following we will apply this general algorithm into two simple examples:

Example A

Suppose we have $N$ identical copies of spin $1 / 2$ all prepared in the same but unknown pure quantum state. If we chose the group $G$ to be $U(2)$, i.e. the complete unitary group transforming a two-level quantum system, we can straightforwardly apply the optimal estimation scheme as described above. To be more precise, due to the fact that there exist elements of the group $U(2)$ for which the reference state is the fixed point (i.e., it is insensitive to its action) we have to work only with the coset space $\left.{ }^{S U(n)}\right|_{U(n-1)}[5]$. In the present case this is a subset of the $S U(2)$ group parameterized by two Euler angles $\theta, \psi$ (the third Euler angle $\chi$ is fixed and equal to zero). This subset is isomorphic to the Poincare sphere.

The unitary representation $U$ is now the representation $\left(\frac{1}{2}\right)$ (we use a standard classification of $S U(2)$ representations, where $(j)$ is the spin number). Its $N$-fold symmetrized direct product (we denote this representation as $U^{N}$ ) is the representation classified as $\left(\frac{N}{2}\right)$ (which transforms a spin- $N / 2$ particle). Choosing the standard basis $|j, m\rangle$ with $m=-j, \ldots j$ in which the coordinate expression for $U(\theta, \psi)$ corresponds to standard rotation matrices $D_{m, n}^{j}(\theta, \psi, 0)=\mathrm{e}^{-i m \psi} d_{m, n}^{j}(\theta)$ [8], we obtain the matrix expression for the operator $\hat{F}$

$$
\begin{aligned}
F_{m, n} & =\int_{0}^{2 \pi} d \phi \int_{0}^{\pi} \frac{\sin (\theta) d \theta}{8 \pi}(1+\cos \theta) \\
& \times D_{m, \frac{N}{2}}^{\frac{N}{2}}(\theta, \phi) D_{n, \frac{N}{2}}^{\frac{N}{2} *}(\theta, \phi)=\frac{N / 2+m+1}{(N+2)(N+1)} \delta_{m, n} .
\end{aligned}
$$

When we insert this operator in the Eq.(5) we immediately find the upper bound on the mean fidelity to be equal to $\frac{N+1}{N+2}$. This is the result derived by Massar and Popescu [1] who have also shown that this upper bound can be attained using the special POVM which consists of an infinite continuous set of operators proportional to isotropically rotated projector $\left|\frac{N}{2}, \frac{N}{2}\right\rangle\left\langle\frac{N}{2}, \frac{N}{2}\right|[9]$.

However, following our algorithm, we can now construct the optimal POVM which is finite. To do so, we have to find a finite set of pairs of angles $\left\{\left(\theta_{r}, \psi_{r}\right)\right\}$ such that the completeness conditions (8) which now take the form

$$
\sum_{r} c_{r}^{2} \mathrm{e}^{-i \psi_{r}(m-n)} d_{m, \frac{N}{2}}^{\frac{N}{2}}\left(\theta_{r}\right) d_{n, \frac{N}{2}}^{\frac{N}{2}}\left(\theta_{r}\right)=\delta_{m, n}
$$

are fulfilled. Following our general scheme we first satisfy the completeness conditions (11) for diagonal terms [compare with Eq.(9)]

$$
\sum_{r} c_{r}^{2} d_{m, \frac{N}{2}}^{\frac{N}{2}}\left(\theta_{r}\right)^{2}=1 ; \quad m=-N / 2, \ldots N / 2 .
$$

To satisfy these completeness conditions we choose $N+1$ angles $\theta_{r}$ to be equidistantly distributed in the $\langle 0, \pi\rangle$ (obviously, there are many other choices which may suite the purpose - see discussion below Eq.(9)). Then we solve the system of linear equations for $N+1$ variables $c_{r}^{2}$. For this choice of $\theta_{r}$ the system (12) has non-negative solutions. Finally we satisfy the off-diagonal conditions by choosing $N+1$ angles $\psi_{s}=\frac{2 s \pi}{N+1}$ for each $\theta_{r}$. In this case $\frac{1}{N+1} \sum_{s=0}^{N} \mathrm{e}^{i \psi_{s} y}=\delta_{y, 0}$ for all $y=-N / 2, \ldots N / 2$ and the off-diagonal conditions are satisfied straightforwardly. This concludes the construction of the optimal and finite POVM for the spin-1/2 state estimation.

\section{Example B}

The algorithm can also be used to estimate a unitary evolution of quantum systems. Consider, for example, a system of $N$ qubits, all initially prepared in some reference state and undergoing a free evolution described by the $U(1)$ group (e.g. like in the Ramsey type experiments 10). Our task is to find a measurement which provides the optimal estimation of the phase of the $U(1)$ rotation. N.B. the phase estimation without any a priori information is different from the frequency standards 
experiments, where the issue is the ability to distiguish neighbouring quantum states with the best resolution (see, for example [1]).

All unitary irreducible representations of $U(1)$ are one dimensional and they are parameterized by a single integer number $(j)$. Rotation of a single isolated qubit is described by a representation $U$ classified as $(0) \oplus(1)$ (here $\oplus$ denotes a direct sum of representations). The entire system of $N$ qubits (these are assumed to be nonentangled) is then transformed due to the representation $U^{N}$ which is equal to the symmetrized $N$-fold direct product of the basic representation $U$. The representation $U^{N}$ is equal to a direct sum of irreducible representations of the form $(0) \oplus(1) \oplus \ldots(N)$ which act in the $N+1$ dimensional space spanned by basis vectors $|m\rangle, m=0,1, \ldots N$. In this basis matrix elements $\hat{F}_{m, n}$ of the operator $\hat{F}$ given by Eq.(4) take the form

$$
\begin{aligned}
\hat{F}_{m, n} & =\int_{0}^{2 \pi} \frac{d \psi}{2 \pi} \frac{\sqrt{\left(\begin{array}{l}
N \\
N-m
\end{array}\right)\left(\begin{array}{l}
N \\
N-n
\end{array}\right)}}{2^{N+1}} \mathrm{e}^{i \psi(n-m)}(1+\cos \psi) \\
& =\frac{\sqrt{\left(\begin{array}{l}
N \\
N-m
\end{array}\right)\left(\begin{array}{l}
N \\
N-n
\end{array}\right)}}{2^{N+2}}\left(2 \delta_{m, n}+\delta_{m, n+1}+\delta_{m+1, n}\right)
\end{aligned}
$$

The upper bound on the fidelity Eq. (5) is now too conservative to be of any use (greater than unity). We can, however, solve the system of Eqs. (9) which in this particular case reads

$$
[\hat{F}-\hat{L}]|\Psi\rangle=0, \quad|\langle m \mid \Psi\rangle|^{2}=1 ; \quad \forall m .
$$

The condition $\operatorname{Det}(\hat{F}-\hat{L})=0$ now determines the eigenvector $|\Psi\rangle$ with the zero eigenvalue as a function of Lagrange multipliers $L_{m}$. When we substitute this eigenvector into the second equation in Eq.(14) we obtain a set of equations for $L_{m}$ from which the reference state $|\Psi\rangle$ can be determined. The final POVM is then constructed by rotation of $|\Psi\rangle$ by $N+1$ angles $\phi_{s}$ in such a way that all off-diagonal elements of $\sum_{s}\left(\hat{O}_{s}\right)_{m, n}$ become equal to zero. This is done in exactly the same way as in Example A. The resulting POVM corresponds to the von Neumann measurement performed on the composite system of all $N$ ions.

We note that the mean fidelity $\bar{f}$ has a rapidly growing number of local extrema which originate from the roots of certain polynomials. From this set of extrema we easily choose the global maximum which corresponds to the optimal POVM.

The maximal mean fidelity for the first six $N$ is: $\frac{3}{4}, \frac{2+\sqrt{2}}{4}, \frac{11+2 \sqrt{3}}{16}, \frac{5+\sqrt{6}}{8}, \frac{32+5 \sqrt{2}+2 \sqrt{5}+5 \sqrt{2} \sqrt{3+2 \sqrt{2}}}{64}$, $\frac{32+10 \sqrt{3}+3 \sqrt{10}+\sqrt{6}}{64}, \ldots$ which approximately gives: 0.750 , $0.854,0.904,0.931,0.947,0.957, \ldots$.

In conclusion, we have presented a general algorithm for the optimal state estimation from finite ensembles. It provides finite POVMs which, following the Neumark theorem [12], can, at least in principle, be implemented as simple quantum computations.
We thank Serge Massar, Susana Huelga, Thomas Pellizzari and Chiara Macchiavello for helpful discussions. This work was supported by the Open Society Fund and FCO, the United Kingdom EPSRC, European TMR Network ERP-4061PL95-1412, Hewlett-Packard, ElsagBailey, The Royal Society, and the Grant Agency VEGA of the Slovak Academy of Sciences.

[1] S. Massar and S. Popescu, Phys. Rev. Lett. 74, 1259 (1995)

[2] A. Peres, Quantum Theory: Concepts and Methods (Kluwer Academic Publishers, Dodrecht, 1993)

[3] C.W. Helstrom, Quantum Detection and Estimation Theory (Academic Press, New York, 1976)

[4] We note that this isotropy condition is equivalent to a "no a priori information" condition and is associated with the specific integration measure in Eq.(11). This measure has to be invariant under the action of all unitary transformations on the state space of pure states.

[5] A.O. Barut and R. Raczka, Theory of Group Representations and applications (World Scientific, Singapore, 1986)

[6] In the case when the spectrum of the generator $\hat{X}$ is degenerate, i.e., for some $m$ and $n$ we have $\omega_{m}=\omega_{n}$, then our algorithm is still valid, provided we increase a number of Langrange multipliers in Eq.(9) to account for off-diagonal elements $L_{m n}$ and $L_{n m}$ in the definition of the operator $\hat{L}$ in Eq. (9).

[7] The most general choice of $\hat{O}_{r}$ would be $\hat{O}_{r}=$ $\sum_{i} c_{r, i}^{2}\left|\Psi_{r, i}\right\rangle\left\langle\Psi_{r, i}\right|$. However, from the point of view of optimality of the POVM these operators are always less effective than operators $\hat{O}_{r}=c_{r}^{2} U_{r}^{N}\left|\Psi_{r}\right\rangle\left\langle\Psi_{r}\right| U_{r}^{N \dagger}$ which are proportional to one-dimensional projectors.

[8] R.N. Zare, Angular Momentum (John Wiley, New York, 1988)

[9] We note that this POVM is related to the covariant measurement as discussed in A.S. Holevo, Probabilistic and Statistical Aspects of Quantum Theory (North-Holland, Amsterdam, 1982), p. 163.

[10] N.F. Ramsey, Molecular Beams (Oxford University Press, London 1956).

[11] J.J. Bollinger et al., Phys. Rev. A54, R4649 (1996); S.L. Braunstein and C. Caves, Phys. Rev. Lett. 72, 3439 (1994); S.F. Huelga et al., quant-ph/9707014.

[12] M.A. Neumark, C.R. Acad. Sci. URSS 41, 359 (1943) 